\title{
Dialysis Access Steals and Leads to Limb Ischemia
}

\author{
Supraja Thunuguntla ${ }^{1 *}$, Mohammed Rizwan ${ }^{2}$ and Jose Campo Maldonado ${ }^{3}$ \\ ${ }^{1}$ Department of Internal Medicine, USA \\ ${ }^{2}$ Department of Medicine, Bhaskar Medical College, India \\ ${ }^{3}$ Department of Infectious Disease, Internal Medicine, School of Medicine, USA
}

*Corresponding author: Supraja Thunuguntla, Department of Internal Medicine Residency, University of Texas, Rio Grande Valley- Valley Baptist Medical Center, USA.

Received Date: March 05, 2020

Published Date: April 24, 2020

\begin{abstract}
Dialysis Access Associated Steal Syndrome (DASS) reported incidence is low, (6.2\%) [1]. Symptoms are dialysis induced hand pain, coldness, numbness, sensory loss which can lead to inevitable digital gangrene and amputation if the diagnosis is delayed. Duplex arterial ultrasound and electromyography (EMG) can help differentiate DASS from Ischemic monomelic neuropathy (IMN), a variation of DASS. Treatment options are individualized based on the location of the AV fistula, severity of presentation, presence of anatomical anomalies of involved vasculature. Comprehensive review of literature demonstrates this outcome of finger gangrene in young patients with ESRD is primarily associated with preexisting diffuse vascular disease [2].
\end{abstract}

Keywords: Dialysis Access Steal Syndrome; Ischemic monomelic neuropathy; Limb Ischemia; AV access

Abbreviations: DASS: Dialysis Access Steal Syndrome; EMG: Electromyography; IMN: Ischemic Monomeric Neuropathy; AV: Arteriovenous; ESRD: End-stage Renal Disease; ER: Emergency Room; PAI: Proximalization of Arterial Inflow

\section{Introduction}

Patients needing Hemodialysis require a lifelong sustained strategy for creation and maintenance of their hemodialysis access (AV Access) to minimize complications and preserve functionality. We report a 36-year-oldman who presented with dry gangrene of his fingers after uneventfully receiving Hemodialysis for over a year.

\section{Case Presentation}

A 36-year-old man with a history of diabetes mellitus type 1, hypertension, peripheral vascular disease, ESRD on hemodialysis, non-communicative after multiple cerebrovascular accidents who was brought to the Emergency. Room (ER) by his caregiver and sister who had noticed blackening of the fingers of left hand for over a month. Patient is disabled, lives at a nursing \& rehabilitation center. There was extensive discoloration of the left ring finger and tip of the index finger evident on physical exam with corresponding loss of sensation. The affected area appeared black, hardened and dry to palpation and presented a clean line of demarcation compatible with the clinical diagnosis of dry gangrene [1,2] (Figure
1\&2). Radial pulse was weak to palpation. His AV access was located on the same side as the affected (Finger 3).

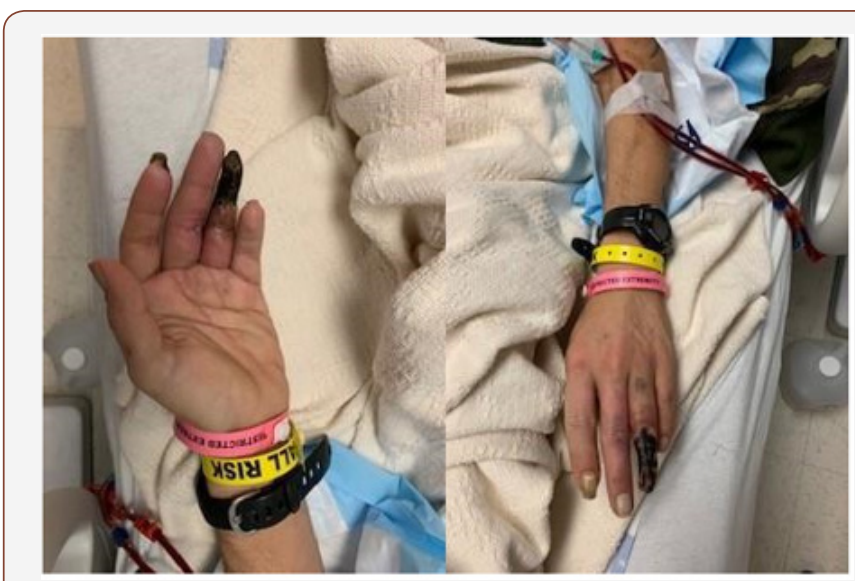

Figure $1 \& 2$ : The line of demarcation is visible on the left ring finger extending to the second phalanx and involving the most distal aspect of the index finger. 


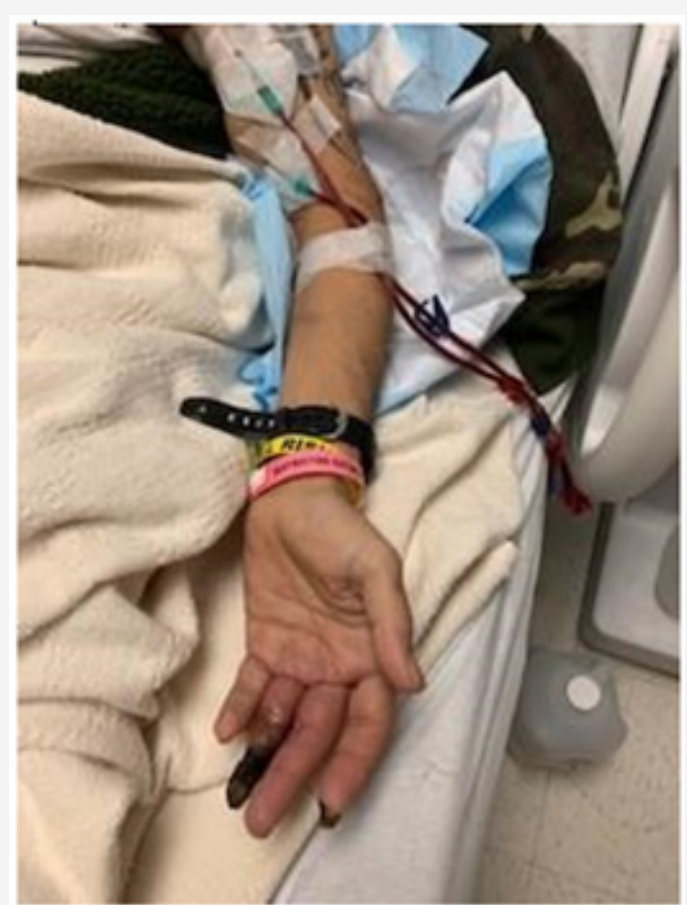

Figure 3: Patient receiving dialysis with the AV access of left arm in use noticed on the same side as the affected hand.

Vital signs on admission were within normal limits. The laboratory work-up revealed hemoglobin of 9.5, glucose level of 480, creatinine level of 5.97. An X Ray of left hand showed dense vascular calcification with thickened arterial wall (Figure 4\&5).

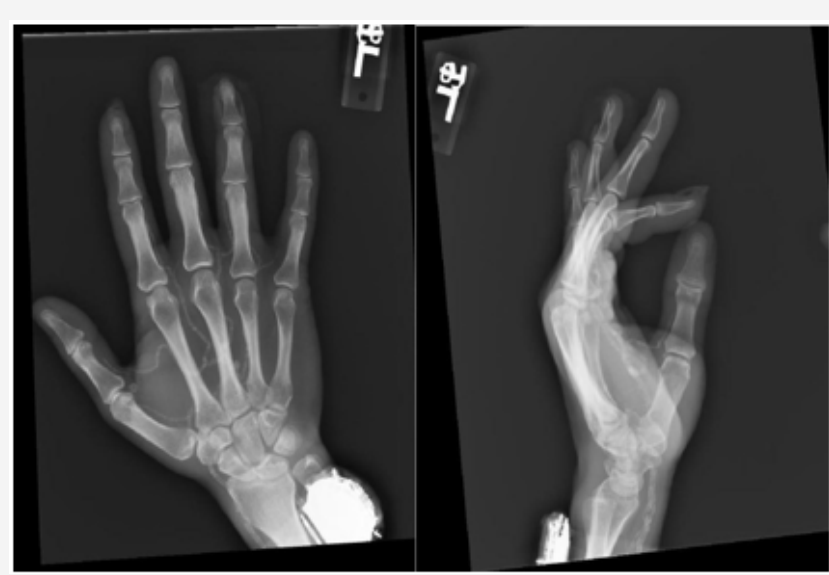

Figure 4 \& 5: Visible calcification of the arterial wall on the $X$ Ray left hand on AP view (4) and lateral (5) view.

Duplex ultrasound of the left upper extremity displayed monophasic waveforms in radial and ulnar arteries. Diffuse calcified arterial walls were present throughout radial and ulnar arteries. Left AV fistula and left brachial artery were difficult to visualize due to post dialysis patches with calciphylaxis- deposition of calcium crystals. The dry gangrene was thought to be an ischemic complication of the arteriovenous (AV) access complicating DASS. Sadly, he presented with advanced disease and irreversible tissue loss (stage IV b). A mainstay of diagnosis is the physical exam findings with supporting duplex ultrasound. DASS was managed by proximalization of arterial inflow (PAI) procedure with preservation of access for future use. During his stay at the hospital, he continued to receive hemodialysis through his left brachial AV fistula. Surgery was complicated by severe bleeding with a hemoglobin drop to 6.0 $\mathrm{g} / \mathrm{dL}$. Amputation of the finger was planned to promote healing and prevent infection of the new graft.

\section{Discussion}

DASS is staged from I to IV based on severity of clinical presentation and propensity to tissue loss, including ischemia presenting with hand pain, poikilothermia, paresthesia, cyanosis or pallor of the digits, and diminished or absent pulses. Pain during dialysis sessions is one of the first symptoms to occur. As our patient is non-communicative, initial symptoms were missed. DASS is also classified depending on timing [3] of symptoms in relation to the AV access, where acute refers to $<24$ hours, within a month or more than a month from AV access creation. Average time of occurrence is in 5 months [4]. This patient had received his brachiobasilic arteriovenous fistula 18 months before. It is important to note the possibility of significant delay in presentation such as in this case. This complication in association with brachiobasilic arteriovenous fistula is relatively high in incidence $(3.7 \%)$ only second to brachiocephalic/basilic AV fistula (5.2 \%) [5]. Risk factors include brachial artery inflow, cerebrovascular disease, hypertension, female gender, coronary artery disease and age above 60 years. Of note, the first three listed were present in our patient. Uncontrolled Insulin dependent diabetes mellitus was present in our patient and is a known major independent risk factor for DASS [6]. There is ambiguity regarding atherosclerotic disease as the primary cause of steal syndrome in young diabetic patients vs due to the hemodialysis $\mathrm{AV}$ access.

Differentiating DASS from Ischemic monomelic neuropathy (IMN) is important in terms of management as the latter often requires aggressive intervention with Arteriovenous access ligation. IMN is caused by focal nerve ischemia affecting the perfusion of the forearm and hand. It is often acute and more common in females. The symptoms are that of nerve palsy with motor dysfunction corresponding to the affected nerve. Peripheral pulses are preserved, and EMG is diagnostic.

Treatment for DASS varies by the stage. Vascular Surgery is frequently indicated for stage IV. The choice of surgery in our patient, PAI is an effort to preserve a functioning hemodialysis AV access and intended to move the inflow for the AV access to a more proximal arterial level, specifically the axillary or proximal brachial artery. Arteriovenous access abandonment with its ligation is indicated for severe functional impairment or impending or frank tissue necrosis.

Prevention is highly difficult as the outcome is difficult to predict. Anticipating and identifying arterial disease avoiding using the brachial artery as an inflow vessel may provide some benefit. Recognition of diffuse atherosclerosis in our patient could have avoided choosing brachial artery as inflow vessel. 


\section{Conclusion}

Knowledge and recognition of this uncommon but devastating condition can help to provide counseling and raise awareness for patients and clinicians potentially avoiding limb loss and function. Surgical intervention when warranted should be aimed at saving the hemodialysis access whenever possible with ligation of the AV access considered a last resource.

\section{Acknowledgement}

\section{None.}

\section{Conflicts of Interest}

No Conflicts of Interest.

\section{References}

1. Davidson D, Louridas G, Guzman R, Tanner J, Weighell W, et al. (2003) Steal syndrome complicating upper extremity hemoaccess procedures: incidence and risk factors. Can J Surg 46: 408-412.
2. Yeager RA, Moneta GL, Edwards JM (2002) Relationship of hemodialysis access to finger gangrene in patients with end-stage renal disease. J Vasc Surg 36: 245-249.

3. Scheltinga MR, Van Hoek F, Bruijninckx CM (2009) Time of onset in haemodialysis access-induced distal ischaemia (HAIDI) is related to the access type. Nephrol Dial Transplant 24: 3198.

4. Kokkosis AA, Abramowitz SD, Schwitzer J, Scott Nowakowski, Victoria J Teodorescu, et al. (2014) Inflow stenosis as a contributing factor in the etiology of AV access-induced ischemic steal. J Vasc Access 15(4): 286290.

5. Zanow J, Petzold M, Petzold K, et al. Diagnosis and differentiated treatment of ischemia in patients with arteriovenous vascular access. In: Vascular Access for Hemodialysis VII, Henry ML (Eds) Gore, pp. 201, Chicago, USA.

6. Davidson D, Louridas G, Guzman R, John Tanner, Wendy Weighell, et al. (2003) Steal syndrome complicating upper extremity hemo access procedures: incidence and risk factors. Can J Surg 46(6): 408-412. 